\title{
EEES, modelo de enseñanza y programa Erasmus en la Escuela de Ingeniería Informática de la ULPGC
}

\author{
Abraham RoDRíGUEz RoDRÍGUEZ \\ Universidad de Las Palmas de Gran Canaria \\ arodriguez@dis.ulpgc.es
}

\begin{abstract}
Resumen
Este trabajo describe el modelo de enseñanza que la Escuela de Ingeniería Informática (EII) utiliza con los estudiantes que realizan estancias de movilidad dentro de programa ERASMUS. La EII pone en práctica medidas integradoras y facilitadoras encaminadas a que los estudiantes puedan llevar a cabo una estancia agradable y de la que puedan sacar el mayor provecho. En su mayoría los estudiantes son tutorizados individualmente, aunque son evaluados con el mismo sistema que el resto de estudiantes. Ha resultado ser una experiencia enriquecedora desde el punto de vista formativo y humano, donde se fomenta la comunicación y el intercambio cultural en el ámbito de la UE.
\end{abstract}

Palabras clave: EEES, ERASMUS, Programación docente, movilidad de estudiantes

\section{EEES, teaching model and ERASMUS program in the EII of the ULPGC}

\begin{abstract}
This work describes the teaching model the EII follows with the students of the ERASMUS mobility program. The EII applies some integration policies to facilitate the integration and outcome of the incoming students during their mobility stays. Although most of the students are tutored individually, they are evaluated using the same marking scheme as the local ones. It has proved to be an enriching experience, from both the training and human points of view, which encourages communication and cultural exchange within the EU.
\end{abstract}

Keywords: EEES, ERASMUS, Course Schedule, students' mobility

\section{Referencia normalizada:}

Rodríguez Rodríguez, A. (2013) EEES, modelo de enseñanza y programa Erasmus en la Escuela de Ingeniería Informática de la ULPGC. Historia y Comunicación Social. Vol. $18 \mathrm{~N}^{\circ}$ Especial Noviembre. Págs. 547-557.

Sumario: 1. Introducción 1.1programa ERASMUS 2. La escuela de ingeniería informática en el EEES 2.1 La EII y la movilidad europea 3. El modelo de enseñanza 4. Análisis del modelo implantado 5. Conclusiones 6. Referencias bibliográficas 


\section{Introducción}

La puesta en marcha del EEES ha permitido avanzar hacia una universidad europea competitiva, lo que conlleva un acercamiento del perfil de la formación a las necesidades reales de los empleadores, mayores oportunidades de trabajo a los egresados, facilidades en la homologación y reconocimiento de títulos a nivel europeo y una promoción en la movilidad de estudiantes y profesores. Estos logros se fundamentan en las bases establecidas por el EEES en la Declaración de Bolonia (Ministros de educación de la UE, 1999), que se traducen en una estructura europea homogénea de títulos de grado y posgrado, con igual valoración de carga lectiva de los diferentes cursos, estudios, asignaturas y calificaciones, y una estructura de formación continua fácilmente entendible por todos los estados miembros.

Dentro del EEES, "los programas de movilidad juegan un papel clave en la política de la UE para la reforma de la educación superior", tal como manifestó el Comisario Europeo de Educación durante la reunión con los ministros de educación de los estados miembro en abril del 2012 (Comisión Europea, 2012). Asimismo, el Comisario considera que "la educación superior debe ser el núcleo de todos los esfuerzos para superar la actual crisis, generando nuevas oportunidades para nuestros jóvenes". De hecho, los ministros de Educación allí reunidos acordaron que los estados miembros deben centrarse en tres objetivos principales de cara a afrontar y superar la crisis económica: proporcionar educación superior de calidad a un mayor número de estudiantes, mejorar la empleabilidad de los estudiantes dotándoles de habilidades mejor consideradas/valoradas en el ámbito empresarial y fortalecer la movilidad de los estudiantes como forma de obtener una mejor formación. Es más, reforzar la movilidad de estudiantes en la UE no solo mejora la empleabilidad de los estudiantes, sino que amplía la colaboración dentro de la UE y fuera de ella.

\subsection{Programa Erasmus}

ERASMUS $^{1}$ es el mayor programa de movilidad de estudiantes de la UE y una de sus iniciativas más conocidas. El programa se estableció en 1987 y desde entonces ya han participado más de 3 millones de estudiantes de 33 países europeos (Comisión Europea, 2013a). El programa ERASMUS, junto con algunos otros programas de formación, se incorporó en 1995 como parte del programa Sócrates de la UE. Éste fue sustituido por el Sócrates II en el 2000, el cual fue posteriormente reemplazado por el Lifelong Learning Programme en el 2007 dando soporte a proyectos conjuntos, redes docentes y escuelas de verano, con el objetivo de mejorar la conexión entre el sistema educativo, el mercado de trabajo y la sociedad como conjunto (Comisión Europea, 2013a).

El programa ERASMUS contó para el curso 2011-12 con un presupuesto de más de 450 millones de euros, facilitando la movilidad de más de 250000 estudiantes en un

1 Portal del Programa ERASMUS: http://ec.europa.eu/education/lifelong-learning-programme/erasmus_en.htm 
solo curso académico, según datos publicados por la EU en su informe anual (Comisión Europea, 2013b). España ha sido tradicionalmente el país receptor de un mayor número de estudiantes (40.000 en el curso 2011/12) seguido por Alemania y Francia, contando con seis universidades en el top 10 europeo de movilidad (Comisión Europea, 2013c). Con las cifras actuales, se estima que aproximadamente el $4,5 \%$ de todos los estudiantes europeos disfrutan de una beca ERASMUS en algún momento de su estudios. El objetivo para el año 2020 sitúa en el $20 \%$ el número de estudiantes que deberán haber cursado parte de sus estudios en el extranjero. (Comisión Europea, 2012). Para el período 2014-2020 la UE ha definido el programa ERASMUS para todos o ERASMUS+, el cual integra diversas iniciativas formativas como el Lifelong Learning Programme y programas exclusivos de la educación superior como Tempus o ERASMUS Mundus. El nuevo programa espera alcanzar unas cifras de movilidad superiores a los 5 millones de personas, contando para ello con un presupuesto global de más de 18.000 millones de euros (Duque García, 2013).

\section{La Escuela de Ingeniería Informática en el EEES}

La ULPGC, una de las universidades pioneras en la puesta en marcha en los años 80 de los estudios de Informática a nivel nacional, cuenta en su Escuela de Ingeniería Informática con una media anual de 800 estudiantes distribuidos entre todas las titulaciones que imparte actualmente (Grado en Ingeniería Informática, Doble Grado en Ingeniería Informática y Administración y Dirección de Empresas, titulaciones propias y títulos en extinción).

La necesaria adaptación de las titulaciones al EEES, según las directrices establecidas en el proceso de Bolonia ha supuesto un gran esfuerzo de adaptación para el Centro y para los profesores que imparten docencia, principalmente debido a la nueva formación basada en competencias que debe adquirir el estudiante y a la distribución curricular de los estudios de informática. Este modelo ha significado un cambio metodológico importante, en la que el estudiante asume un papel proactivo en el proceso de enseñanza-aprendizaje. Los profesores, por el contrario, se convierten en instrumentos del proceso de enseñanza-aprendizaje en los que los estudiantes se apoyan para conseguir las competencias formativas. Como era de esperar, este proceso ha traído consigo múltiples ventajas, desde la necesaria eliminación de algunas materias obsoletas, hasta la creación de nuevas materias, pasando por la actualización de los contenidos de todas las materias que permanecen. El rediseño del sistema de enseñanza de las asignaturas, con nuevos tipos de actividades formativas y un modelo de evaluación basado en la evaluación continua, se cuentan también entre los aspectos positivos a remarcar dentro del nuevo marco de enseñanza. 


\subsection{La EII y la movilidad europea}

La EII participa en el programa ERASMUS casi desde sus orígenes contando en conjunto más de 80 plazas anuales para estudiantes salientes y otras tantas para estudiantes entrantes. Los estudiantes realizan sus estancias en las universidades con las que la EII ha suscrito acuerdos bilaterales, entre las que se encuentran universidades de países como Alemania, Polonia, Francia, Italia, Dinamarca o Noruega, entre otros.

La política del Centro en este campo ha sido siempre favorable a la apertura de nuevos convenios que aumenten la oferta para nuestros estudiantes y profesores. La relación con las universidades socias siempre ha tendido a estrechar lazos de colaboración facilitando la recepción de estudiantes fuera de cupo o la extensión del período de enseñanza, siempre que no supongan una carga adicional importante para el sistema. En línea con la política de cordialidad con las universidades socias, el control sobre la elaboración de los acuerdos académicos de los estudiantes entrantes recae principalmente sobre los coordinadores de origen, actuando la EII como asesor académico del estudiante.

Tabla 1. $\mathrm{N}^{\mathrm{o}}$ estudiantes de movilidad recibidos en la EII por año y país de origen.

\begin{tabular}{|r|c|c|c|c|c|c|}
\hline \multicolumn{1}{|c|}{ País/curso } & $\mathbf{0 8 / 0 9}$ & $\mathbf{0 9 / 1 0}$ & $\mathbf{1 0} / \mathbf{1 1}$ & $\mathbf{1 1 / 1 2}$ & $\mathbf{1 2 / 1 3}$ & Total \\
\hline Polonia & 1 & 10 & 18 & 23 & 13 & 65 \\
\hline Alemania & & & 3 & 1 & 3 & 7 \\
\hline Italia & 7 & 7 & 3 & 5 & 5 & 27 \\
\hline Francia & & 1 & 0 & 3 & 1 & 5 \\
\hline Eslovenia & & & 2 & 1 & 3 & 6 \\
\hline República Checa & 2 & 4 & 3 & 3 & 3 & 15 \\
\hline Otros & 2 & 4 & 1 & 0 & 5 & 12 \\
\hline Total & 12 & 26 & 30 & 36 & 33 & 137 \\
\hline
\end{tabular}

La Tabla 1 muestra la distribución de los estudiantes entrantes por principales nacionalidades en los últimos cinco cursos académicos. A partir del curso 08/09 hemos observado un cambio en la tendencia, con crecimientos significativos en los siguientes años, llegándose a triplicar el número de estudiantes entrantes en tan sólo tres cursos académicos. Dicho aumento se suele achacar principalmente al crecimiento medio experimentado por el programa ERASMUS a nivel europeo, que ha sido de un $7 \%$ para el caso español y ligeramente inferior $(6,1 \%)$ para el conjunto de los países participantes. En el caso de la EII, gran parte de este crecimiento también se debe a la labor de promoción exterior realizada por el Centro, estableciendo nuevos convenios con diversas universidades en los que se ponía en valor las cualidades del Centro y de su entorno socioeconómico. Esta política se complementó con acciones de información al profesorado del Centro al objeto de recabar su colaboración en la recepción de estudiantes extranjeros. 
Con este contexto, se estrecharon lazos con varias universidades, especialmente polacas, ya que se consideraron estratégicamente relevantes para nuestros estudiantes salientes por varios motivos. Por un lado, dichas universidades nos han garantizado siempre la impartición de un número significativo de materias en inglés, lo cual es sin duda interesante de cara a que los estudiantes puedan sacar provecho real de las asignaturas cursadas en el extranjero. Por otro lado, al tratarse de un país con un nivel de vida asequible permitía que un mayor número de estudiantes pudiese beneficiarse de los programas de movilidad, dada la escasa cuantía de las becas del programa. Este aspecto, aunque no es el objeto de este artículo, permitió que el número de estudiantes salientes de la EII tuviera una progresión similar a la de los estudiantes entrantes. Además de las universidades polacas, que son el origen de aproximadamente el $50 \%$ de los estudiantes que recibe la Escuela, los estudiantes italianos representan el segundo grupo más numeroso de estudiantes entrantes, seguidos a cierta distancia de estudiantes de la República Checa, Alemania y Eslovenia.

En cuanto a la motivación de los estudiantes para elegir nuestro Centro, según nuestra experiencia los estudiantes eligen la EII principalmente debido a factores como la facilidad y el bajo coste de las conexiones de transporte aéreo con prácticamente toda Europa, el clima y la seguridad global del entorno urbano. En nuestra experiencia el atractivo académico y la facilidad a la hora de configurar los acuerdos académicos son generalmente factores secundarios en la elección de los destinos de los estudiantes ERASMUS.

\section{EI modelo de enseñanza}

Desde el punto de vista de los estudiantes, la movilidad ha cobrado un mayor interés dentro del EEES por el mayor atractivo que tienen las titulaciones ya adaptadas al espacio europeo. Por otro lado, la implantación de las directrices de Bolonia ha supuesto la desaparición del sistema de créditos propio de cada país miembro en favor del Sistema Europeo de Transferencia de Créditos (ECTS), simplificando la elaboración de los acuerdos académicos al utilizar la misma unidad como medida del esfuerzo que ha de realizar el estudiante. Otro factor a analizar dentro del contexto lo determina el nivel general de conocimientos de los estudiantes entrantes, tanto del idioma como de las materias. En general, la formación específica que traen los estudiantes que llegan al Centro es buena o muy buena, con buenos fundamentos en informática, excepto en aquellas universidades en las que la titulación no es una informática pura (como puede ser el caso de titulaciones de informática empresarial). La mayoría son estudiantes de últimos cursos de carrera, por lo que buscan materias muy específicas y avanzadas, o la realización de proyectos de fin de título. Por supuesto también existen los casos de estudiantes que buscan simplemente cubrir el número de créditos que le exige su universidad sin importar la materia a cursar, lo cual se percibe en la actitud y predisposición del estudiante. Afortunadamente, este tipo de estudiantes no han sido un grupo numeroso. 
En cuanto al nivel de conocimientos de idiomas, muy pocos estudiantes tienen un nivel de español que les permita seguir la docencia sin problemas, es decir, nivel B2 o superior. Destaca, en general, el buen nivel de inglés de los estudiantes entrantes en prácticamente todas las nacionalidades excepto para el caso de los estudiantes de universidades italianas, aunque, debido a la similitud con el español, no supone un obstáculo determinante. La política institucional de la ULPGC obliga a que el idioma de impartición de la docencia sea el español. Por este motivo, el idioma en el que se imparte casi toda la enseñanza del Centro es el español, a excepción de dos asignaturas de técnicas de comunicación que se imparten en inglés. Este hecho limita y condiciona significativamente el modelo de enseñanza del Centro, en lo que se refiere a la recepción de estudiantes ERASMUS, tal como se analizará en las siguientes secciones.

Un último aspecto contextual a analizar lo constituye la cantidad de carga de trabajo que poseen los estudiantes de movilidad en su estancia en la EII. Los requisitos exigidos sobre el número de créditos matriculados son establecidos por la universidad de origen y, en general, son similares a los que se establecen para los estudiantes salientes, con valores cercanos a los 60 créditos para estancias anuales y 25 créditos para estancias de un semestre (ver Tabla 2). Aunque los datos que obran en nuestro poder indican que sólo el $25 \%$ de los estudiantes entrantes optan por cursar un curso completo en el Centro, la realidad indica que esta tendencia da la vuelta cuando se acerca el momento de volver a casa, siendo muchos (hasta el 80\%) los que optan por extender su estancia al curso completo.

En cuanto a las asignaturas elegidas, los estudiantes se han decantado mayoritariamente por materias 'asequibles' como lo son aquellas relacionadas con los idiomas, y por materias específicas o avanzadas de nivel de máster, como desarrollo web, análisis numérico avanzado o visión por computador. Hay que reconocer sin embargo que algunas de las materias ofertadas han quedado sistemáticamente fuera de las preferencias de los estudiantes, debido principalmente a las limitaciones por parte del profesorado para comunicarse adecuadamente en un idioma extranjero.

El Centro considera que es fundamental para la inserción de los estudiantes el proporcionar un trato individualizado a cada uno de los estudiantes entrantes facilitándoles asistencia en la selección de materias, flexibilizando los períodos de ampliación o modificación de la matrícula, permitiendo modificaciones razonables en los acuerdos académicos y elaborando guías y recursos dirigidos a facilitar la integración y la gestión administrativa de los estudiantes.

Otra de las medidas facilitadoras del Centro consiste en posibilitar que el estudiante entrante se matricule de cualquier materia impartida en el Centro siempre que el estudiante tenga un dominio suficiente del idioma español. En otro caso, en cada curso académico se publica una lista de las materias y profesores que están dispuestos a seguir un modelo diferenciado de enseñanza con los estudiantes, siguiendo el modelo que posteriormente se comentará. 
Tabla 2. Número medio de créditos ECTS matriculados por los estudiantes entrantes, excluyendo a los estudiantes que sólo realizaron la movilidad para realizar el PFC.

\begin{tabular}{|l|c|c|c|c|}
\hline Créditos matriculados/Curso & $\mathbf{0 9 / 1 0}$ & $\mathbf{1 0 / 1 1}$ & $\mathbf{1 1 / 1 2}$ & $\mathbf{1 2 / 1 3}$ \\
\hline En curso completo & 38 & 48 & 60 & 67 \\
\hline En un semestre & 26 & 20 & 25 & 27 \\
\hline En la EII & 23 & 26 & 25 & 23 \\
\hline En otros Centros & 4 & 4 & 10 & 10 \\
\hline
\end{tabular}

También en la línea de facilitar las cosas a los estudiantes entrantes se les permite que la matrícula de materias en otros centros de la ULPGC (ver Tabla 2), lo cual en algunos casos puntuales ha producido situaciones en las que determinados estudiantes 'puentean' al Centro y se matriculan mayoritariamente de asignaturas en otras titulaciones. Por este motivo esta política se ha restringido en los últimos años en la ULPGC, exigiendo una matrícula mínima de materias en el centro de acogida, ya que no tiene mucho sentido que un estudiante haga una estancia con un acuerdo entre centros sin cursar ninguna asignatura en el mismo.

En general el sistema de enseñanza que se propone a los estudiantes entrantes sigue alguna de las siguientes alternativas:

- A) El estudiante entrante se integra como uno más en el aula con los estudiantes locales.

- B) El estudiante es tutorizado individualmente por el profesor que asigna trabajos y proyectos específicos para estudiantes ERASMUS.

- C) El estudiante es tutorizado individualmente por el profesor pero el sistema de evaluación es el mismo que para los estudiantes locales (trabajos, exposiciones, exámenes, etc.), con la salvedad de que se le facilita la elaboración de la actividad en inglés.

La primera opción es seguida por un reducido número de estudiantes (inferior al $15 \%$ ) debido a que apenas hay una oferta formativa en inglés, debido a la política interna de la ULPGC, y a que el propio estudiante no domina suficientemente el idioma español. La opción B era la opción mayoritaria en los planes de estudios pre-Bolonia por lo que actualmente está en desuso. La opción C es la que adoptan la mayoría de los docentes del Centro, ya que es la más equitativa para todos los estudiantes, dentro de las limitaciones que impone la utilización de otra lengua. El seguimiento personalizado a los estudiantes garantiza la posibilidad de realizar evaluación continua a los estudiantes entrantes, proporcionándoles material en inglés que les permita seguir la asignatura y realizar las mismas actividades (o equivalentes) a las que realizan sus compañeros españoles.

Qué duda cabe que este sistema de enseñanza personalizado supone una carga de trabajo adicional para los docentes, que además no está reconocido dentro del 
cómputo de la carga docente de la ULPGC. Es gracias a este trabajo adicional y desinteresado que los estudiantes reciben una formación adecuada y obtienen un buen rendimiento de la estancia que realizan en nuestro Centro.

\section{Análisis del modelo implantado}

En general, el rendimiento obtenido por los estudiantes es bueno, si consideramos las características específicas de la movilidad, el área de conocimiento de la titulación y la complejidad de las materias que usualmente cursan en la EII. La Tabla 3 muestra el porcentaje de superación de los créditos con respecto a los créditos matriculados por los estudiantes, recogiendo únicamente las materias efectivamente cursadas por el estudiante, es decir, eliminando renuncias y cambios en los acuerdos académicos. El rendimiento para los estudiantes que sólo cursaron un semestre es ligeramente superior al de los estudiantes que cursaron el curso completo, alcanzando cifras que rondan el $80 \%$ para materias cursadas en la EII (sobre 20 créditos ECTS) y del $90 \%$ (sobre 6 créditos ECTS, mayoritariamente) para las matriculadas en otros centros, siendo en este caso, 'Español para extranjeros' la asignatura de otros centros incluida mayoritariamente en el acuerdo académico.

Tabla 3. Media de rendimiento académico de los estudiantes entrantes en términos de créditos superados frente a créditos matriculados, excluyendo a los estudiantes que sólo realizaron la movilidad para realizar el PFC

\begin{tabular}{|l|c|c|c|c|}
\hline Rendimiento/Curso & $\mathbf{0 9} / \mathbf{1 0}$ & $\mathbf{1 0} / \mathbf{1 1}$ & $\mathbf{1 1} / \mathbf{1 2}$ & $\mathbf{1 2 / 1 3}$ \\
\hline Créditos superados de la EII & $80 \%$ & $66 \%$ & $75 \%$ & $74 \%$ \\
\hline Créditos superados de otros Centros & $84 \%$ & $81 \%$ & $79 \%$ & $80 \%$ \\
\hline
\end{tabular}

Si bien el rendimiento académico obtenido por los estudiantes es más fue aceptable, tal como recoge la Tabla 3, hemos detectado que la integración de los estudiantes extranjeros con los locales es muy baja a pesar de las medidas integradoras puestas en marcha por parte de la ULPGC y de la EII. No cabe duda de que tenemos que trabajar en mejorar este punto ya que esta falta de sinergia limita el enriquecimiento del entorno educativo de las instituciones receptoras, tal como establece uno de los principales objetivos de la movilidad ERASMUS.

Entre los aspectos positivos que valoran los estudiantes entrantes de la EII destacan la accesibilidad y buena predisposición del profesorado y de los responsables de movilidad en el Centro, el alto contenido práctico de la docencia, la disposición y eficiencia de los servicios administrativos de la Escuela, y la comprensión de las circunstancias específicas de cada caso, especialmente en lo referente a la utilización del inglés en las actividades y trabajos académicos y a los plazos de entrega de los mismos. 
Por otro lado, prácticamente la totalidad de estudiantes entrantes demandan año tras año la impartición de un número significativo de créditos en inglés, acompañado cada vez más de un aumento de la oferta formativa de materias avanzadas y actuales. No cabe duda de que la impartición de materias en inglés posee numerosas ventajas de valor añadido entre las que podríamos citar:

- Mejora de la imagen exterior del Centro y de la Universidad.

- Limitar las incidencias entre el profesorado y el alumnado, debidas en muchos casos a la barrera del idioma.

- Simplificar los procesos de elaboración/ratificación de los acuerdos académicos, ya que se orientaría a la mayoría del alumnado a la oferta formativa en inglés.

- Facilitar la creación de nuevos convenios con universidades que imparten docencia en inglés para nuestros estudiantes y que exigen como condición sine qua non una política de reciprocidad en la oferta de materias en inglés para los estudiantes que nos envían.

- Para los estudiantes locales que cursen las asignaturas supondría una incuestionable mejora de sus habilidades de idiomas antes de iniciar su programa de movilidad o como complemento formativo.

- Por último, serviría como elemento motivador para animar a nuestros estudiantes a participar en los programas de movilidad.

Por su parte la EII, en coordinación con otros Centros de la ULPGC, ha solicitado que se le permita la impartición de 60 créditos ECTS en inglés, y se encuentra actualmente elaborando diversos títulos propios al objeto de aumentar y mejorar la oferta formativa del Centro. Tanto la ULPGC como la EII están tomando algunas medidas encaminadas a conseguir los objetivos establecidos en el nuevo programa ' $E R A S$ MUS para todos', entre los que destacan los planes formativos y cursos de perfeccionamiento de inglés dirigidos al profesorado, o los cursos de idiomas semestrales para estudiantes entrantes. Para completar estas medidas, y tal como se recoge en el nuevo programa de movilidad, los Centros de universidades diferentes a lo largo y ancho de Europa deberían trabajar en la elaboración de planes conjuntos (join-degrees) que desarrollen completamente y hagan realidad el EEES.

\section{Conclusiones}

En esta comunicación ha descrito el modelo de enseñanza que se sigue en la Escuela de Ingeniería Informática (EII) de la Universidad de Las Palmas de Gran Canaria como receptor de estudiantes del programa de movilidad ERASMUS, dentro del EEES. La UE considera que el proceso de Bolonia debe conducir hacia un Espacio Europeo de Educación Superior (EEES) en el que los estudiantes en movilidad puedan elegir cursos y asignaturas de alta calidad y beneficiarse de procedimientos de reconocimiento de créditos sencillos y transparentes. La EII tiene una larga tradi- 
ción en la movilidad de estudiantes que participan en programas de movilidad tanto SICUE (en el territorio nacional) como ERASMUS (dentro de la Unión Europea). En los últimos 5 años, cerca de 200 estudiantes nacionales y del resto de Europa han realizado estancias en el Centro, con una media anual cercana a los 40 estudiantes.

Considerando las características actuales de la EII en cuanto a dimensionamiento y oferta formativa, el número de estudiantes que se reciben se encuentra dentro de los parámetros medios del programa ERASMUS para el periodo actual (un 4,5\% de estudiantes). Este volumen se ha conseguido gracias al esfuerzo de la EII y de los profesores que la forman, que han mostrado una extraordinaria comprensión hacia las especificidades de los estudiantes extranjeros. Aun así, es evidente que se deben tomar nuevas medidas encaminadas a aumentar el número de estudiantes que se reciben, en consonancia con las perspectivas descritas en el nuevo programa 'ERASMUS para todos 2014-2020', el cual estima que, al menos, se dupliquen los beneficiarios de la iniciativa ERASMUS-formación.

En una escuela como la EII, con una media anual de 800 estudiantes, la llegada de 40 estudiantes que se incorporan mayoritariamente a las asignaturas de los últimos cursos puede ocasionar situaciones en las que más del $50 \%$ de los estudiantes sean extranjeros. En esos casos es donde se ponen a prueba las habilidades comunicativas y humanas de los elementos intervinientes en el proceso de enseñanza-aprendizaje, dado que ni siquiera el elemento básico del proceso, la comunicación, está garantizado. Hay que tener en cuenta que se producen dificultades de comunicación derivadas de la falta de conocimiento del idioma local por parte de los estudiantes extranjeros y también por la limitación de algunos profesores al expresarse en inglés. Detectadas estas debilidades, tanto la EII como la propia universidad han luchado por superarlas fomentando la realización de cursos de español para los estudiantes extranjeros y de inglés para los profesores del Centro. Otra de las debilidades detectada ha sido la dificultad de implantar el modelo de evaluación continua, que el EEES requiere, en asignaturas donde las actividades que se programan, y los recursos disponibles, están en español en su mayoría. A este problema se le ha intentado dar la vuelta programando actividades en inglés, inclusive para los estudiantes locales. Por otro lado, el profesorado también se implica realizando tutorizaciones personalizadas en inglés a los estudiantes extranjeros que más dificultad tienen con el español.

No cabe duda de que el proceso implica también muchas ventajas. Desde el punto de vista del profesor se produce un enriquecimiento al recibir estudiantes de otras culturas, al tener la posibilidad de desarrollar sus habilidades comunicativas en otros idiomas, al poder contrastar los niveles de conocimiento y capacitación entre los estudiantes locales y extranjeros, y al poder comparar programas de aprendizaje. En lo que a los estudiantes se refiere, las ventajas vienen derivadas del intercambio cultural, de la motivación que produce trabajar en grupos multiculturales (y en ocasiones multidisciplinares) y del acercamiento a culturas extranjeras que despiertan el deseo de completar la formación en el extranjero. Por parte del Centro las ventajas se traducen en una promoción de la imagen corporativa en el extranjero, elemento positivo que permite captar futuros estudiantes desde esos países. 
En resumen, la recepción de estudiantes extranjeros ha resultado ser una experiencia enriquecedora, tanto desde el punto de vista formativo como humano, donde se fomenta la comunicación y el intercambio cultural en el ámbito de la UE.

\section{Referencias bibliográficas}

Publicaciones web

COMISIÓN EUROPEA (2013a). Number of ERASMUS students tops 3 million. Disponible en http://europa.eu/rapid/press-release_IP-13-657_en.htm. [27-102013].

COMISIÓN EUROPEA (2013b) ERASMUS programme in 2011-12: the figures explained. Disponible en http://europa.eu/rapid/press-release_MEMO-13-647 en.htm. [27-10-2013].

COMISIÓN EUROPEA (2013c). ERASMUS - Statistics. Disponible en http://ec.europa.eu/education/erasmus/statistics_en.htm. [27-10-2013].

COMISIÓN EUROPEA (2012). Higher education reform key for jobs and growth, Bologna Process Ministers are told. Disponible en http://europa.eu/rapid/press-release IP-12-394 en.htm?locale=en. [27-10-2013].

DUQUE GARCÍA, M. (2013): ERASMUS+. Organismo Autónomo Programas Educativos Europeos. Disponible en http://www.oapee.es/oapee/inicio/ErasmusPlus.html. [27-10-2013].

MINISTROS DE EDUCACIÓN DE LA UNIÓN EUROPEA (1999): The Bologna Declaration of 19 June 1999. Disponible en: http://www.eees.es/pdf/Declaracion Bolonia.pdf [27-10-2013].

\section{El autor}

Abraham Rodríguez Rodríguez es Licenciado en Informática y Doctor en Informática por la Universidad de Las Palmas de Gran Canaria (ULPGC). Actualmente ocupa una plaza de profesor Titular de Universidad que está adscrita al Departamento de Informática y Sistemas de la ULPGC, donde ha estado 23 años impartiendo docencia en asignaturas del ámbito de Ciencias de la Computación e Inteligencia Artificial. Ha ocupado diversos cargos de gestión en la ULPGC, entre ellos el de Subdirector de Relaciones Internacionales de la Escuela de Ingeniería Informática durante los años 2007 a 2012. 